\title{
Modeling the Coordinated Operation between Bus Rapid Transit and Bus
}

\author{
Jiaqing Wu, ${ }^{1,2}$ Rui Song, ${ }^{1}$ Youan Wang, ${ }^{1}$ Feng Chen, ${ }^{3}$ and Shubin $\mathrm{Li}^{4}$ \\ ${ }^{1}$ School of Traffic and Transportation, Beijing Jiaotong University, Beijing 100044, China \\ ${ }^{2}$ Beijing Public Transport Holdings (Group) Co., Ltd., Beijing 100161, China \\ ${ }^{3}$ Beijing Transportation Research Center, Beijing 100161, China \\ ${ }^{4}$ Public Security Department, Shandong Police College, Jinan 250014, China
}

Correspondence should be addressed to Rui Song; rsong@bjtu.edu.cn

Received 25 July 2014; Accepted 3 November 2014

Academic Editor: Huimin Niu

Copyright (C) 2015 Jiaqing Wu et al. This is an open access article distributed under the Creative Commons Attribution License, which permits unrestricted use, distribution, and reproduction in any medium, provided the original work is properly cited.

\begin{abstract}
The coordination between bus rapid transit (BRT) and feeder bus service is helpful in improving the operational efficiency and service level of urban public transport system. Therefore, a coordinated operation model of BRT and bus is intended to develop in this paper. The total costs are formulated and optimized by genetic algorithm. Moreover, the skip-stop BRT operation is considered when building the coordinated operation model. A case of the existing bus network in Beijing is studied, the proposed coordinated operation model of BRT and bus is applied, and the optimized headway and costs are obtained. The results show that the coordinated operation model could effectively decrease the total costs of the transit system and the transfer time of passengers. The results also suggest that the coordination between the skip-stop BRT and bus during peak hour is more effective than non-coordination operation.
\end{abstract}

\section{Introduction}

In modern metropolises like Beijing, the demand of mass transit has increased rapidly. Both bus rapid transit (BRT) and bus system play a role in alleviating the urban traffic congestion and pollution problems. Comparing BRT with bus system, BRT system is a kind of transportation with larger capacity and higher punctuality rate. However, bus is more flexible, because it can run on more roads rather than the separated bus lane. Thus, the coordinated operation of BRT and bus can maximize their strengths. The coordinated operation of BRT and feeder bus systems is very important for an integrated mass transit system, which can improve both operation efficiency and service level of the urban mass transit system.

So far, many attempts have been made to solve the feeder bus network design and scheduling problems. The first and important step to solve the problem is network design for feeder bus [1]. Therefore, researchers studied the network design problem using various pieces of methods [2-7]. Once the network is determined, the bus scheduling problems are the most important part in operation. Wirasinghe et al. [8] determined optimal transit service characteristics that minimize the sum of the transit operating costs and passenger time costs for a coordinated rail and bus transit system. Shrivastava et al. [9] applied genetic algorithm for solving the problems of scheduling and schedule coordination. Ngamchai and Lovell [10] proposed a new model showing how genetic algorithms could be manipulated to help optimize bus transit routing design, incorporating unique service frequency settings for each route. In addition, Ting and Schonfeld [11] used a heuristic algorithm to optimize the headways and slack times for all coordinated routes and minimized the total costs of operating a multiple-hub transit network.

The previous review suggests that studies mainly focus on the routing and scheduling problems, especially on the cooperation between railway and bus. Fewer studies focus on the coordinated operation between BRT and its feeder bus. However, with the rapid development of BRT and bus 
in big cities in China, it is necessary to study the coordinated operation problems of BRT and bus. Therefore, a coordinated operation model of BRT and bus is established by analyzing the operation characteristics of the BRT and bus system in this paper. Considering that skip-stop BRT operation can reduce operation costs and passengers' in-transit travel times without extra technological improvement, the skipstop operation of BRT is included in the model, which is not considered in previous studies. In order to solve the model and improve the computing efficiency, genetic algorithm is applied in the paper. Finally, the proposed coordinated operation model is applied in a real network of BRT in Beijing. The minimum system cost is achieved by using genetic algorithm to solve the coordinated operation model. The results are significant for the construction and operation of the integrated public transport.

\section{Coordinated Operation Model of BRT and Bus}

The objective of the coordinated operation model of BRT and bus is to minimize the total costs, including bus operation costs and the passenger costs. The operation costs include wages and welfare cost of drivers and daily vehicle operation and maintenance costs. The costs of passengers contain waiting costs, transfer costs, and in-vehicle costs. Then the coordinated operation model of BRT and bus is formulated.

2.1. Operation Costs. Operation costs $\left(C_{o}\right)$ contain the driver's salary and welfare cost $\left(C_{p}\right)$ and the daily operation and maintenance costs of vehicles $\left(C_{b}\right)$. The mathematical formulation is expressed as follows:

$$
C_{o}=C_{p}+C_{b}
$$

where the driver's salary and welfare cost is the product of unit cost of driver driving time and vehicle operating time:

$$
C_{p}=\alpha_{p} \cdot \sum_{i=1}^{i=n} f_{i} \cdot T_{i}
$$

where $\alpha_{p}$ is unit cost of driver driving time, $f_{i}$ is frequency of BRT or bus, $T_{i}$ is vehicle operating time, and $n$ is the number of the BRT lines and bus lines.

Daily vehicle maintenance costs contain the fixed cost and variable cost. The equation is expressed as follows:

$$
C_{b}=\sum_{i=1}^{i=n} f_{i} \cdot L_{i} \cdot\left(c_{0}+c_{1} \cdot N_{i}\right)
$$

where $L_{i}$ is the route length $(\mathrm{km}), c_{0}$ is fixed cost (yuan $\left./ \mathrm{km}\right)$, $c_{1}$ is variable cost (yuan $/ \mathrm{km} \cdot \mathrm{seat}$ ), and $N_{i}$ is the seats number of a vehicle.

2.2. Passenger Costs. Passenger costs contain waiting cost, transfer cost, and in-vehicle cost of passengers.
2.2.1. Waiting Costs. Waiting costs are the costs passengers spend on the stop waiting for their first bus during their trips. Assuming that the system consists of a BRT line and $n$ bus lines, the expectation for passengers' waiting time is half interval of the bus when the bus arrival interval is uniform. Then the waiting cost can be formulated as follows:

$$
C_{W}=C_{W B}+C_{W b}=\left(\sum_{i, j \in S} \frac{H_{B} q_{o i j B}}{2}+\sum_{b=1}^{n} \sum_{i, j \in S} \frac{H_{b} q_{o i j b}}{2}\right) \cdot \alpha_{w},
$$

where $S$ is the stop set, $H_{B}, H_{b}$ are BRT and bus interval (min), $q_{o i j B}$ is passenger flow from stop $i$ to stop $j$ (people), and $\alpha_{w}$ is the unit cost of passenger waiting time (yuan/min).

2.2.2. Transfer Costs. Transfer costs are caused by transfer time when passengers transfer from a BRT stop/bus stop to a bus stop/BRT stop. The time includes walking time and transfer waiting time. Generally, transfer walking time is a constant linked to the distance between the platforms. In addition, the transfer waiting time relates to its feeder bus's arrival time distribution at the transfer stop. In the system consisting of a BRT line and a number of buses lines, passengers mainly transfer from BRT to bus or from bus to BRT. Thus, the passenger transfer costs can be calculated as follows:

$$
C_{T}=C_{B b}+C_{b B}=\left(\sum T_{B b} q_{B b}+\sum T_{b B} q_{b B}\right) \cdot \alpha_{t}
$$

where $T_{B b}$ and $T_{b B}$ are the transfer time for passengers transferring from BRT stop/bus stop to bus stop/BRT stop (min), $q_{B b}$ and $q_{b B}$ are passenger flow transferring from BRT stop/bus stop (people), and $\alpha_{t}$ is the unit cost of passenger transfer time (yuan/min).

Combined with the existing operation mode of BRT, allstop operation of BRT is widely used. However, during morning and evening peak hour, the skip-stop operation of BRT has become an important way to improve the operational efficiency. Both the all-stop and skip-stop operations are considered during the peak hour to improve the operational efficiency and decrease the total costs. Therefore, transfer time at peak hour and off-peak hour is calculated separately.

\section{(1) Transfer Time at Peak Hour}

(a) Passengers Transfer from the BRT Stop to the Bus Stop at All-Stop Stop. The minimum successful transfer time for passengers transferring from a BRT stop to a bus stop is $t_{B \text { min }}=t_{B o}+t_{w}-s_{b} . t_{B o}$ is the time for passengers getting off from the BRT vehicle ( $\mathrm{min}), t_{w}$ is the time for passengers walking to the BRT stop ( $\mathrm{min}$ ), and $s_{b}$ is the time for the bus stopping at the stop ( $\mathrm{min}$ ). If a passenger transfers from $t$ bus stop to a BRT stop successfully, $t_{b}-t_{B} \geq t_{B \text { min. }} \cdot t_{b}$ and $t_{B}$ are arrival time of the bus and BRT. Therefore, the successful expected transfer time is formulated as follows:

$$
T_{B b}=\int_{-H}^{H} f\left(t_{B}\right) d t_{B} \int_{t_{B}=t_{B \min }}^{H+\Delta t} f\left(t_{b}\right)\left(t_{b}-t_{B}\right) d t_{b},
$$


where $\Delta t$ is slack time of bus (at transfer point of the line, the total costs of system allow the vehicle to arrange some "slack" time to reduce the failed probability of transfer).

(b) Passengers Transfer from the BRT Stop to the Bus Stop at Stop-Skip Stop. During the morning and evening peak hour, except for the all-stop BRT line, skip-stop operation will be used at some larger transfer stop to convey passengers. Now the minimum transfer time from the BRT to the bus is $t_{B \text { min }}^{\prime}=\min \left(t_{B o}+t_{w}-s_{b}, t_{S B o}+t_{w}-s_{b}\right) . t_{S B o}$ is the time for passengers getting off from a skip-stop BRT vehicle. Thus, the condition for passengers transferring from the BRT to the bus successfully is $t_{b}-t_{B} \geq t_{B \min }^{\prime}$. Therefore, the successful expected transfer time for passengers transferring from BRT to bus is formulated as follows:

$$
T_{B b}=\int_{-H}^{H} f\left(t_{B}\right) d t_{B} \int_{t_{B}=t_{B \min }^{\prime}}^{H+\Delta t} f\left(t_{b}\right)\left(t_{b}-t_{B}\right) d t_{b} .
$$

(c) Passengers Transfer from the Bus Stop to the BRT Stop at All-Stop Stop. The minimum successful transfer time for passengers transferring from the bus stop to the BRT stop is $t_{b \min }=t_{b o}+t_{w}-s_{B} \cdot t_{b o}$ is the time for passengers getting off from the bus (min), and $s_{B}$ is the time for the BRT stopping at the stop ( $\mathrm{min}$ ). If a passenger transfers from a bus stop to a BRT stop successfully, $t_{B}-t_{b} \geq t_{b \text { min }}$. Therefore, the success expected transfer time is formulated as follows:

$$
T_{b B}=\int_{-H-\Delta t}^{H-\Delta t} f\left(t_{b}\right) d t_{b} \int_{t_{b}=t_{b \min }}^{H} f\left(t_{B}\right)\left(t_{B}-t_{b}\right) d t_{B} .
$$

(d) Passengers Transfer from the Bus Stop to the BRT Stop at Stop-Skip Stop. During the peak hour, the skip-stop BRT alleviates the pressure to convey a large number of passengers effectively at the large transfer stop and increases the successful transfer probability for passengers at the same time. At the moment, the minimum transfer time from the bus to the BRT is $t_{b \text { min }}^{\prime}=\min \left(t_{b o}+t_{w}-s_{B}, t_{b o}+t_{w}-s_{S B}\right)$. Then the condition for passengers transferring from the bus to the BRT successfully is $t_{B}-t_{b} \geq t_{b \text { min }}^{\prime}$. Therefore, the successful expected transfer time for passengers transferring from bus to BRT is formulated as follows:

$$
T_{b B}=\int_{-H-\Delta t}^{H-\Delta t} f\left(t_{b}\right) d t_{b} \int_{t_{b}=t_{b \min }^{\prime}}^{H} f\left(t_{B}\right)\left(t_{B}-t_{b}\right) d t_{B} .
$$

(2) Transfer Time at Off-Peak Hour. The skip-stop BRT operation is not applied during off-peak hour. Then the expected transfer time is calculated as previous (a) and (c) during off-peak hour.

According to the previous discussion, if transfer passenger volumes and passenger transfer time costs are known, the transfer costs can be achieved after calculating the expected transfer time.

2.2.3. In-Vehicle Costs. In-vehicle costs are caused by the time passengers spent in the bus. At the same bus system, the passenger time costs are similar, and in-vehicle costs relate to the operating time and passenger volume of the operating section. In-vehicle costs can be calculated as follows:

$$
C_{I}=\left(\sum_{i, j \in S} \sum_{l=1}^{n} t_{l i j} q_{l i j}+\sum_{i, j \in S} t_{i j} q_{i j}\right) \cdot a_{o},
$$

where $t_{l i j}$ and $t_{i j}$ are the operating time (min) from stop $i$ to stop $j$ of bus and BRT, $q_{l i j}$ and $q_{i j}$ are the passenger volume (people) for the section $i$ to $j$ of bus and BRT, and $a_{o}$ is the unit cost of passenger in-vehicle time.

2.3. Mathematical Formulation of the Coordinated Operation Model of BRT and Bus. To minimize the total costs of coordinated operation of BRT and its feeder bus, the model can be expressed as follows:

$$
\begin{array}{ll}
\min & C=C_{O}+C_{U}=C_{O}+C_{W}+C_{T}+C_{I} \\
\text { s.t. } & H_{B} \leq \frac{60 P_{B} \beta_{B}}{q_{B \max }} \\
& H_{b} \leq \frac{60 P_{b} \beta_{b}}{q_{b \max }} \\
& \leq \Delta t<\frac{H}{2},
\end{array}
$$

where $P_{B}$ and $P_{b}$ are total vehicle capacity (people) of a BRT vehicle and a bus, $\beta_{B}$ and $\beta_{b}$ are maximum allowable load factors of a BRT vehicle and a bus, and $q_{B \text { max }}$ and $q_{b \text { max }}$ are the passenger volume (people/h) of the maximum section of passenger flow of the BRT and the bus. Besides, $C_{O}$ and $C_{U}$ in the model can be obtained from Sections 2.1 and 2.2.

\section{Genetic Algorithm Based Solution for the Proposed Model}

The proposed model for BRT and its feeder bus is a nonlinear programming model with single objective and multiple variables. A deterministic analytical method cannot be used for such a model. However, the heuristic algorithm, such as genetic algorithm, can solve the problem effectively. In addition, from the literature review it is evident that heuristic approaches have been very popular for solving problems. These methods typically are examined when a specific problem is created by a mathematical formula. Heuristic methods have been used by Kuah and Perl [2] to quickly search for approximately good solutions by means of different sets of rules to generate routes in step-by-step and iterative procedures. Shrivastav and Dhingra [12] developed a heuristic algorithm applied in the model for integrating the suburban stops and bus services. Besides, Chowdhury and Chien [13] proposed a model seeking for better coordination of the intermodal transit system. Kuan et al. [14] proposed the design and analysis of two metaheuristics, genetic algorithms and ant colony optimization, for solving the feeder bus network design problem. Shrivastava and O'Mahony [15] used genetic algorithms to develop feeder routes and frequencies leading to schedule coordination of feeder buses with main 
transit simultaneously. Therefore, the genetic algorithm is used in this paper. The steps of genetic algorithm are shown as follows.

(1) Set the parameters of genetic algorithm based on the objective function and constrains of the coordinated operation model, including the population size $M$, the number of variables $n$, crossover probability $P_{c}$, mutation probability $P_{m}$, and stop evolution algebra $T$.

(2) Establish a regional scanner, according to the constraint condition (Formulation 12) of the coordinated operation model of BRT and bus. The variable range is set at the same time.

(3) Generate initial populations randomly in the variable range of step (2). Then the initial total costs are calculated by fitness function.

(4) Implement selection operation using proportional selection operator.

(5) Perform crossover operation in accordance with the crossover probability $P_{c}$. There is an average number of $P_{c} \cdot S$ chromosome (the headway of BRT and bus system) crossing every time for each population.

(6) In order to maintain the population diversity, define $P_{m}$ as the mutation probability of genetic system. There is an average number of $P_{m} \cdot S$ chromosome (the headway of BRT and bus system) mutating each time. Chromosome from $i=1$ to $S$ repeats the following process: generate a random number $r$ from the interval $[0,1]$, if $r \leq P_{m}$, rebuild the chromosome $i$, and test its feasibility. If it is feasible, replace the original chromosome with a new chromosome.

(7) Decode each individual to the real value and calculate local optimal solution.

(8) Calculate the fitness value of each individual of the local optimal solution set, and save the best headway of BRT and bus system.

(9) Evaluate whether the stop evolution algebra $T$ is satisfied. If it is satisfied, the calculation results are outputted. If not, return to step (4).

\section{A Case Study of the Network Analysis}

In order to verify the effectiveness of the coordinated model, a real transit network is analyzed in the paper. Considering the data availability and the field investigation, BRT Line 1 in Beijing and its 8 feeder buses are chosen as the case study. The transit network is shown as in Figure 1.

4.1. Parameters Setting. As shown in Figure 1, numbers 117 are the bus stops of BRT line 1, and stops 1-17 are the transfer stops. Lines 1-8 are the feeder bus lines of BRT line 1. According to the real operational data, the basic parameters of the BRT line 1 and its feeder bus lines are assumed as follows: total vehicle capacity of a BRT vehicle and a bus is 160 and 90 people. The maximum allowable load factor of a BRT vehicle and a bus is $120 \%$. The passenger volume (people/h) of each section during peak hour 7:00-8:00 and off-peak hour 13:00-14:00 of the BRT line and the bus line is obtained from the smart card data. And the transfer passenger volumes, transfer walking time, and dwell time of BRT and bus are also investigated. Thus, the maximum departure interval is calculated based on Formulation 12 and the maximum section of passenger flow. Besides, unit cost of driver driving time $\alpha_{p}=10.14$ yuan $/ \mathrm{min}$, fixed cost per kilometer $c_{0}=1.5$ yuan $/ \mathrm{km}$, and unit variable cost $c_{1}=$ $0.08 \mathrm{yuan} / \mathrm{km} \cdot$ seat are used in calculating the operation costs. The value of time of passenger is $1.5 \mathrm{yuan} / \mathrm{min}$. Compared with the bus, BRT is a kind of transportation with higher punctuality rate, which runs on the exclusive bus lane. Thus, the arrival time distribution of the BRT vehicle is assumed to be a uniform distribution. And the arrival time distribution of the bus is normal distribution as a result of the uncertainty in the process of operation.

Then genetic algorithm is used to solve the coordinated operation model of BRT and bus. Parameters of genetic algorithm are set as follows:

$$
\begin{aligned}
& \text { population size } M=20, \\
& \text { crossover probability } P_{c}=0.90, \\
& \text { mutation probability } P_{m}=0.07, \\
& \text { number of generations } T=1000 .
\end{aligned}
$$

4.2. Results Analysis. After setting the parameters, the genetic algorithm is applied. The generation is shown as in Figure 2, which shows that the convergence occurred well within 300 generations. The solution continuously improved with the increase of the generation number.

The optimization results of off-peak hour and peak hour are shown in Tables 1 and $2 . h_{B}$ and $h_{1-8}$ represent the optimized headways of BRT line 1 and its 8 feeder buses. (1), (2), (3), and (4) represent four kinds of operation modes. (1) is the coordinated operation of BRT and bus during offpeak hour. (2) is the coordinated operation of all-stop BRT and bus during peak hour. (3) is the coordinated operation of all-stop BRT, skip-stop BRT, and bus during peak hour. (4) is noncoordinated operation of BRT and bus during peak hour.

In order to compare the costs change between different modes, rate of change is used in the paper. The rate is calculated as follows:

The rate of change between (2) and (3)

$$
=\frac{\text { the cost of (2) }- \text { the cost of (3) }}{\text { the cost of (2) }} \text {, }
$$

The rate of change between (2) and (4)

$$
=\frac{\text { the cost of (4) }- \text { the cost of (2) }}{\text { the cost of (4) }} \text {. }
$$

The comparison results between different operation modes are shown in Table 3. 


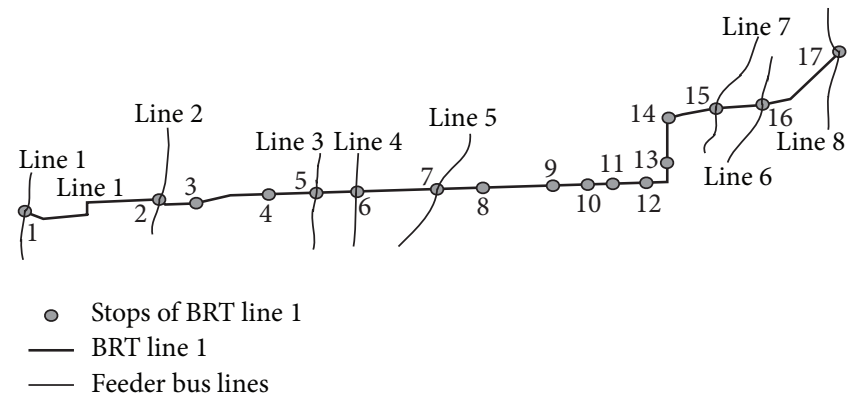

FIGURE 1: The transit network of BRT line 1 and its feeder bus lines.

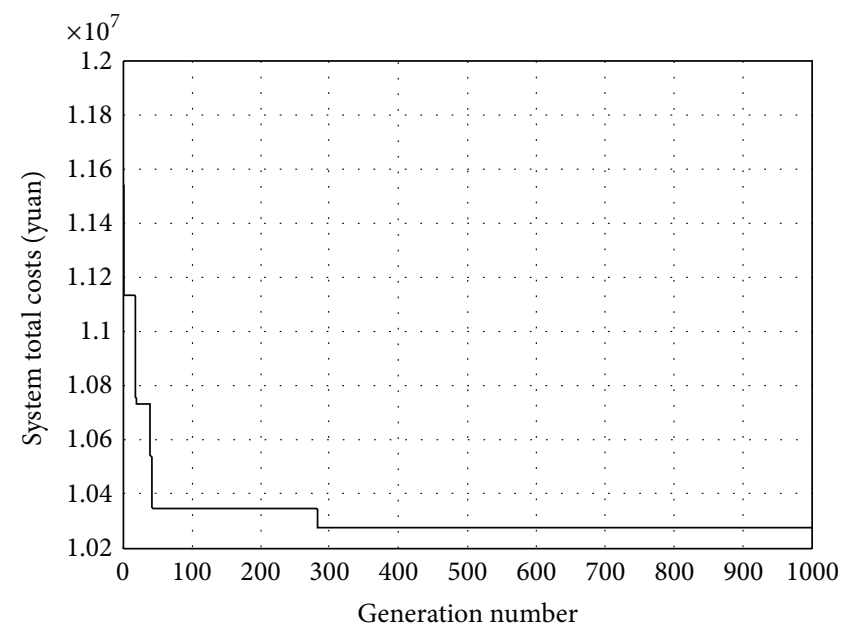

_ Objective function value

(a) The generation of off-peak hour

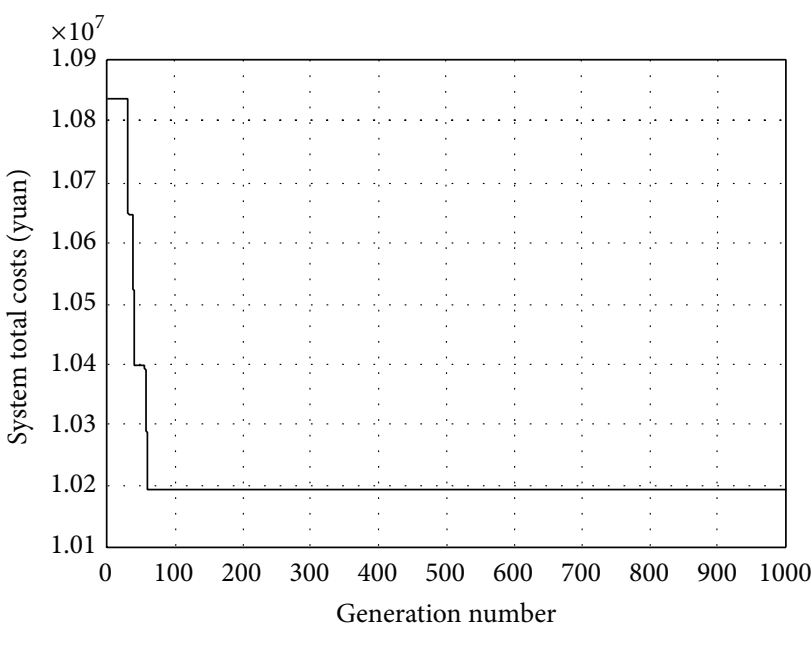

_ Objective function value

(b) The generation of peak hour

FIgURE 2: The generation history of system total costs.

TABLE 1: Optimization results of different operation modes.

\begin{tabular}{|c|c|c|c|c|c|c|c|c|c|}
\hline Headway & & (1) & (2) & (3) & (4) & Slack time & (1) & (2) & (3) \\
\hline \multirow{9}{*}{$\begin{array}{l}\text { Headway } \\
(\min )\end{array}$} & $\mathrm{h}_{\mathrm{B}}$ & 8.07 & 2.19 & $\begin{array}{c}3.83 \text { (skip-stop) } \\
3.86 \text { (all-stop) }\end{array}$ & 2.17 & \multirow{9}{*}{$\Delta t(\min )$} & 3.81 & 2.67 & 3.96 \\
\hline & $\mathrm{h}_{1}$ & 22.50 & 11.69 & 6.41 & 11.85 & & 3.81 & 2.67 & 3.96 \\
\hline & $\mathrm{h}_{2}$ & 20.44 & 12.80 & 11.03 & 12.78 & & 3.81 & 2.67 & 3.96 \\
\hline & $\mathrm{h}_{3}$ & 13.77 & 10.40 & 6.67 & 5.54 & & 3.81 & 2.67 & 3.96 \\
\hline & $\mathrm{h}_{4}$ & 12.31 & 10.25 & 22.98 & 10.73 & & 3.81 & 2.67 & 3.96 \\
\hline & $\mathrm{h}_{5}$ & 16.11 & 7.78 & 8.33 & 10.15 & & 3.81 & 2.67 & 3.96 \\
\hline & $\mathrm{h}_{6}$ & 14.50 & 6.66 & 8.62 & 12.35 & & 3.81 & 2.67 & 3.96 \\
\hline & $\mathrm{h}_{7}$ & 20.42 & 9.10 & 9.94 & 8.02 & & 3.81 & 2.67 & 3.96 \\
\hline & $\mathrm{h}_{8}$ & 23.89 & 10.81 & 11.77 & 11.86 & & 3.81 & 2.67 & 3.96 \\
\hline
\end{tabular}

TABLE 2: Optimization costs of different operation modes.

\begin{tabular}{|c|c|c|c|c|c|}
\hline \multirow{2}{*}{ Operation mode } & \multirow{2}{*}{ Operation costs (yuan) } & \multicolumn{3}{|c|}{ Passenger costs (yuan) } & \multirow{2}{*}{ Total costs (yuan) } \\
\hline & & Waiting costs & Transfer costs & In-vehicle costs & \\
\hline (2) & 7365.4 & 514480 & 5654.6 & 9666500 & 10194000 \\
\hline (3) & 7681.7 & 616760 & 239658.3 & 5844100 & 6708200 \\
\hline (4) & 7249.8 & 526410 & 13440.2 & 9727900 & 10275000 \\
\hline
\end{tabular}


TABLE 3: Comparison results between different operation modes.

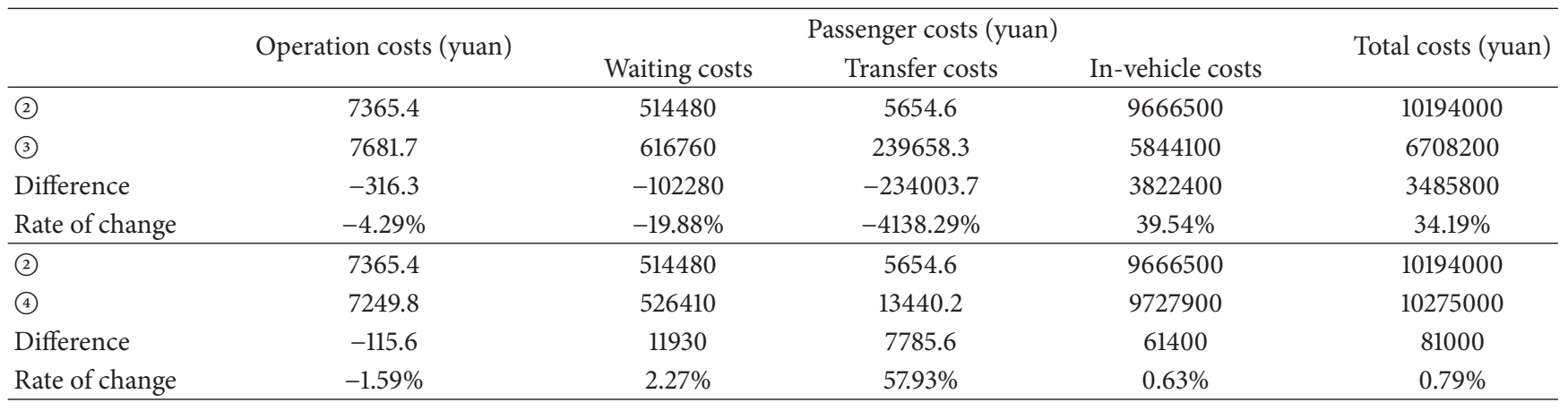

\section{Conclusions}

Route generation and schedule coordination are two important aspects for the operational integration of public transport modes. This study focuses on schedule coordination and a coordinated operation model is established. Then genetic algorism is applied for solving the model effectively. The following conclusions can be drawn from the model development and results analysis.

(1) Optimized headways of the BRT and bus networks can be obtained through the coordinated operation model. Total costs decrease after implementing coordinated operation.

(2) Coordinated operation can improve the efficiency of transfer significantly. Comparing the coordinated and noncoordinated operation of all-stop BRT and bus during peak hour, passenger costs reduce significantly during peak hour through coordinated operation. It is because passengers waiting time decreases when coordinated operation of all-stop BRT and bus is implemented. However, the operation costs increase caused by the larger number of vehicles needed after implementing coordinated operation.

(3) In the paper, coordinated operation model is applied in the operation of both all-stop BRT and skip-stop BRT during peak hour. Comparing the coordinated operation of all-stop BRT and bus during peak hour and the coordinated operation of all-stop BRT and skip-stop BRT and bus during peak hour, the total costs of the system with skip-stop operation decrease significantly. The reason is that, with skip-stop operation, the total travel time becomes shorter than the one for all-stop operation, depending on the stopping constraints. In-vehicle travel time becomes shorter due to skipping stops, although waiting and transfer times increase. However, the operation costs increase due to the larger vehicle fleets after implementing the skip-stop operation. Only when the stopping stops are carefully coordinated can skip-stop service benefit passengers and transit agencies.

\section{Conflict of Interests}

The authors declare that there is no conflict of interests regarding the publication of this paper.

\section{Acknowledgments}

The authors appreciate the financial support of this work that was provided by the National Natural Science Foundation of China (71471104) and the University of Science and Technology Program Funding Projects of Shandong Province (J14LI02).

\section{References}

[1] M. H. Almasi, S. Mirzapour Mounes, S. Koting, and M. R. Karim, "Analysis of feeder bus network design and scheduling problems," The Scientific World Journal, vol. 2014, Article ID 408473, 10 pages, 2014.

[2] G. K. Kuah and J. Perl, “The feeder-bus network-design problem," Journal of the Operational Research Society, vol. 40, no. 8, pp. 751-767, 1989.

[3] M. Hadi Baaj and H. S. Mahmassani, "Hybrid route generation heuristic algorithm for the design of transit networks," Transportation Research C: Emerging Technologies, vol. 3, no. 1, pp. 31-50, 1995.

[4] P. Shrivastav and S. L. Dhingra, "Development of feeder routes for Suburban railway stations using heuristic approach," Journal of Transportation Engineering, vol. 127, no. 4, pp. 334-341, 2001.

[5] S. Chien, Z. Yang, and E. Hou, "Genetic algorithm approach for transit route planning and design," Journal of Transportation Engineering, vol. 127, no. 3, pp. 200-207, 2001.

[6] A. Pradhan and G. Mahinthakumar, "Finding all-pairs shortest path for a large-scale transportation network using parallel Floyd-Warshall and parallel Dijkstra algorithms," Journal of Computing in Civil Engineering, vol. 27, no. 3, pp. 263-273, 2013.

[7] V. M. Tom and S. Mohan, "Transit route network design using frequency coded genetic algorithm," Journal of Transportation Engineering, vol. 129, no. 2, pp. 186-195, 2003.

[8] S. C. Wirasinghe, V. F. Hurdle, and G. Newell, "Optimal parameters for a coordinated rail and bus transit system," Transportation Science, vol. 11, no. 4, pp. 359-374, 1977.

[9] P. Shrivastava, S. L. Dhingra, and P. J. Gundaliya, "Application of genetic algorithm for scheduling and schedule coordination problems," Journal of Advanced Transportation, vol. 36, no. 1, pp. 23-41, 2002. 
[10] S. Ngamchai and D. J. Lovell, "Optimal time transfer in bus transit route network design using a genetic algorithm," Journal of Transportation Engineering, vol. 129, no. 5, pp. 510-521, 2003.

[11] C.-J. Ting and P. Schonfeld, "Schedule coordination in a multiple hub transit network," Journal of Urban Planning and Development, vol. 131, no. 2, pp. 112-124, 2005.

[12] P. Shrivastava and S. L. Dhingra, "Development of coordinated schedules using genetic algorithms," Journal of Transportation Engineering, vol. 128, no. 1, pp. 89-96, 2002.

[13] S. M. Chowdhury and S. I.-J. Chien, "Intermodal transit system coordination," Transportation Planning and Technology, vol. 25, no. 4, pp. 257-287, 2002.

[14] S. N. Kuan, H. L. Ong, and K. M. Ng, "Solving the feeder bus network design problem by genetic algorithms and ant colony optimization," Advances in Engineering Software, vol. 37, no. 6, pp. 351-359, 2006.

[15] P. Shrivastava and M. O’Mahony, “A model for development of optimized feeder routes and coordinated schedules: a genetic algorithms approach," Transport Policy, vol. 13, no. 5, pp. 413425, 2006. 


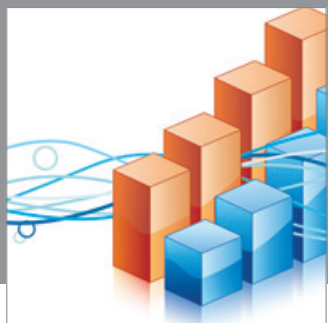

Advances in

Operations Research

mansans

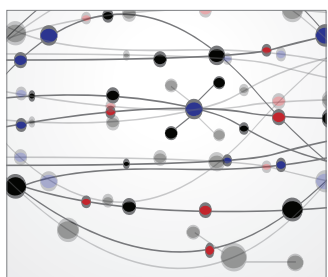

The Scientific World Journal
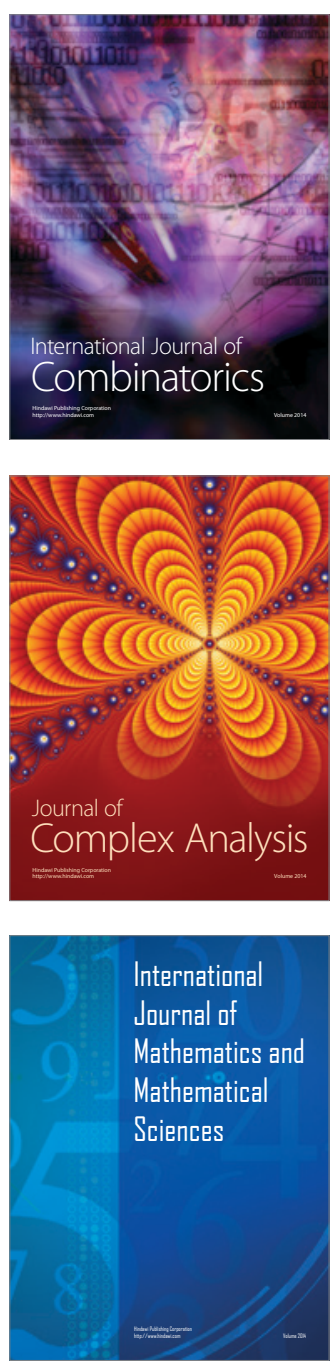
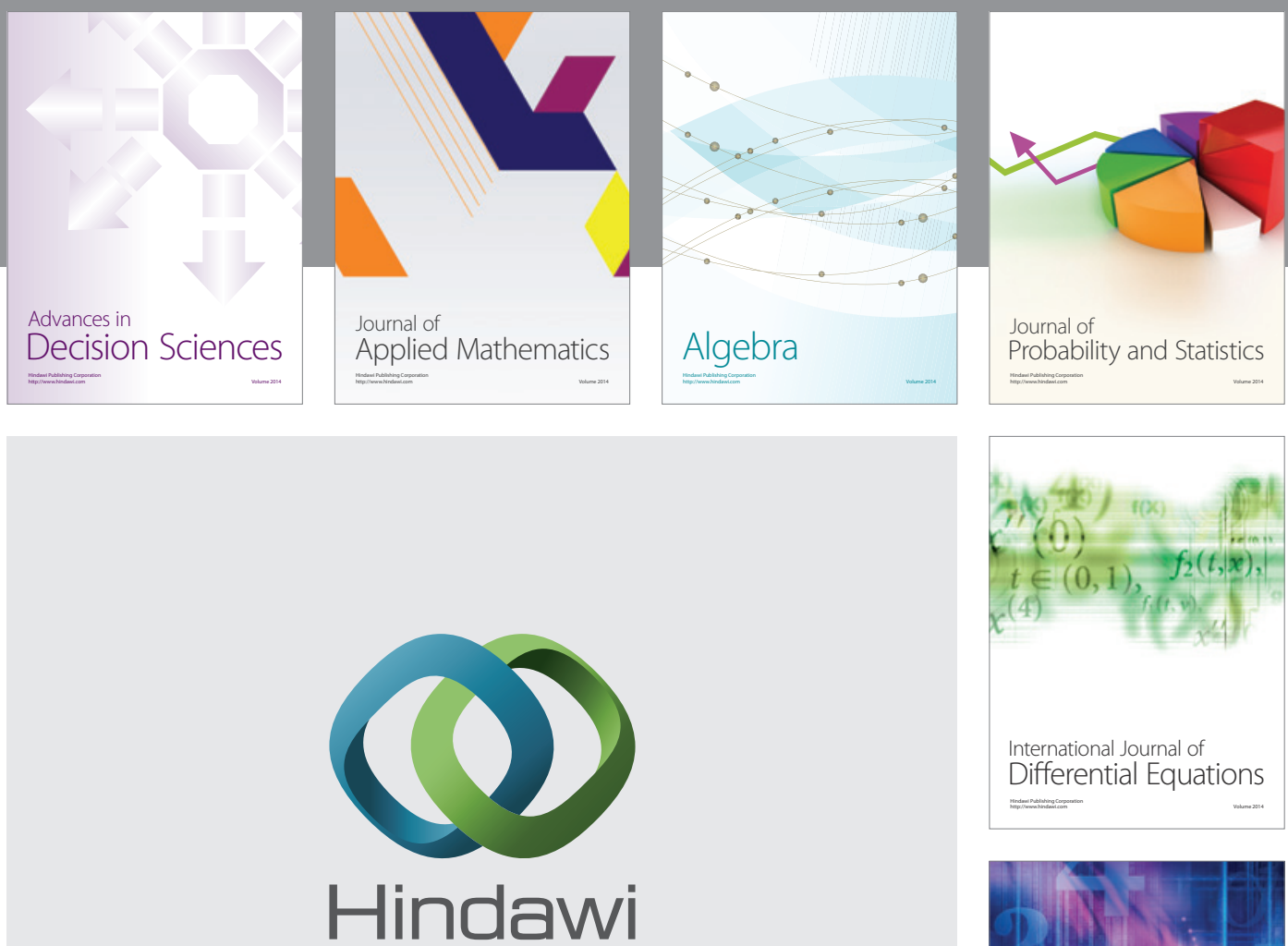

Submit your manuscripts at http://www.hindawi.com
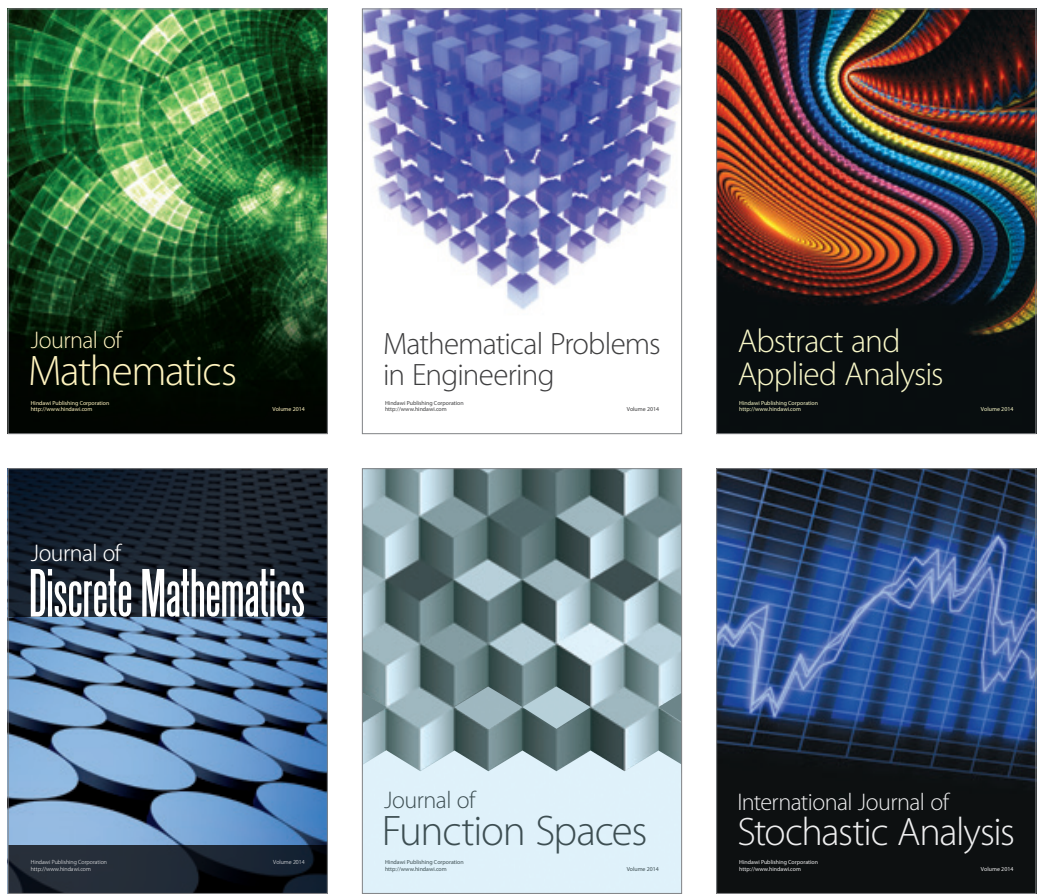

Journal of

Function Spaces

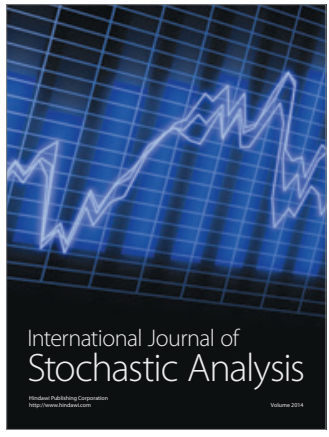

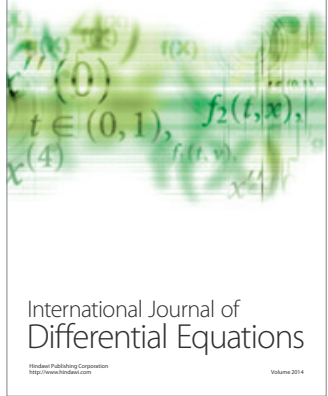
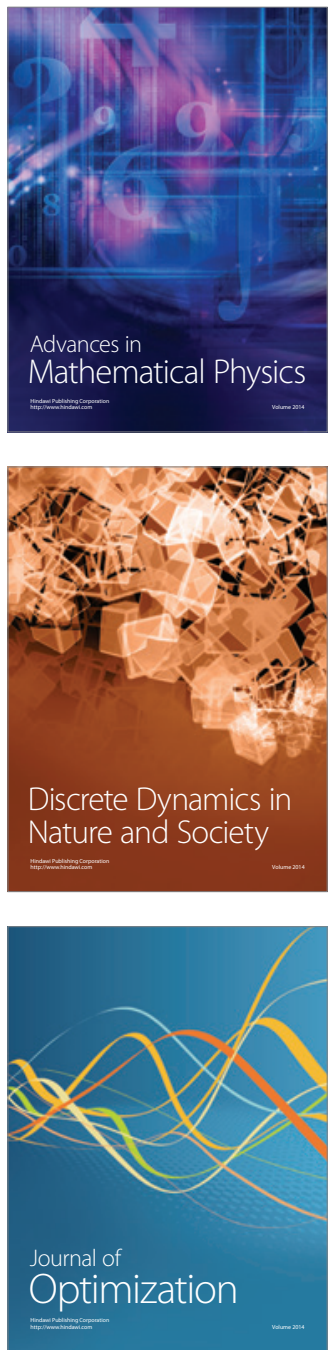